\title{
Scientific Considerations for an In Vitro Bioequivalence Approach for Ultrasound Contrast Agent Products: How Size Distribution and Total Volume of Microbubbles Affect Its Echogenicity
}

\section{Authors}

Bonhye $\mathrm{Koo}^{1,2}$, Bin Qin ${ }^{1}$, Yunbo Liu ${ }^{3}$, Yong Wu${ }^{2}$, Stephanie Choi ${ }^{1}$, Darby Kozak ${ }^{1}$, Jiwen Zheng ${ }^{2}$

Affiliation

${ }^{1 .}$ Division of Therapeutic Performance, Office of Research and Standards, Office of Generic Drugs, Center for Drug Evaluation and Research, Food and Drug Administration, Silver Spring, MD 20993, United States

2. Division of Biology, Chemistry and Materials Science, Office of Science and Engineering

Laboratories, Center for Devices and Radiological Health, Food and Drug Administration, Silver Spring, MD 20993, United States

${ }^{3 .}$ Division of Applied Mechanics, Office of Science and Engineering Laboratories, Center for Devices and Radiological Health, Food and Drug Administration, Silver Spring, Maryland 20993, United States

Ultrasound contrast agents (UCAs), typically a suspension of gas-filled lipid microspheres (microbubbles), are injected intravenously to improve contrast in suboptimal echocardiograms. There is a general correlation between acoustic signal efficiency, ultrasound frequency, as well as the size and concentration of microbubbles [1]. Because the performance of these products is not directly correlated with the concentration profile of the microsphere gas in the blood, developing and approving a generic UCA product based on traditional pharmacokinetic studies is challenging. To address this challenge, alternative methods are needed to assess the equivalence of generic UCA products based on an understanding of the critical quality attributes with appropriate in vitro tests. Moreover, size distribution of UCA microbubbles need to be noted for safety concerns, for example, microbubbles smaller than 10 $\mu \mathrm{m}$ are preferred to prevent micro pulmonary embolism.

In our study, cryogenic scanning electron microscopy (cryo-SEM) micrographs was used to visualize and measure the microbubble size with minimal distortion. The cryo-SEM micrograph revealed the hollow structure of a model UCA (approved sulfur hexafluoride lipid microbubbles) that was originally filled with gas (Figure 1a), with size distribution ranging from $1-10 \mu \mathrm{m}$, along with a rare presence of microbubbles $>10 \mu \mathrm{m}$ (indicated with an arrow in Figure 1a). This was further confirmed using quantitative Coulter Counter measurement which reveals 95\% (number weighted) of the microbubbles $<$ $2 \mu \mathrm{m}$ and $3-6 \mu \mathrm{m}$-sized microbubbles contributing to $48 \%$ of the total bubble volume. When left open to the atmosphere, the concentration of microbubbles decreased by about $22 \%$ in the first hour and then was constant for the next four hours (Figure 1b). This decrease was $14 \%$ for small microbubbles $(<2 \mu \mathrm{m})$, but $52 \%$ for larger microbubbles $(>3 \mu \mathrm{m})$, indicating that larger microbubbles are less stable than smaller microbubbles. To investigate the correlation between size distribution of microbubble formulation and acoustic efficacy, the microbubble suspension was fractionated, and each fraction was subsequently characterized for backscattered coefficient at three clinically relevant frequencies (i.e., 2.5 $\mathrm{MHz}, 5 \mathrm{MHz}$, and $7 \mathrm{MHz}$ ). A linear correlation between the volume concentration of microbubbles and backscattering coefficient was observed at each of the tested frequencies (Figure 2). Findings from this study provide a greater understanding of the critical quality attributes of microbubbles which may be used to support demonstrating the equivalence of a generic UCA product to the reference listed product. 


\section{References:}

[1] J-M Gorce, M Arditi, and M Schneider, Investigative Radiology 35 (2000), p. 661 - 671.

[2] The authors (Bonhye Koo and Bin Qin) are supported in part by an appointment to the Oak Ridge Institute for Science and Education (ORISE) research participation program at the Center for Drug Evaluation and Research (CDER) administered by the ORISE through an agreement between the U.S. Department of Energy and CDER.

[3] The authors would like to acknowledge the FDA Advanced Characterization Facility, Center for Drug Evaluation and Research/Office of Pharmaceutical Quality/Office of Biotechnology

Products/Division of Biotechnology Review and Research II, Center for Devices and Radiological Health/Office of Science and Engineering Laboratories/Division of Applied Mechanics, and Center for Devices and Radiological Health/Office of Science and Engineering Laboratories/Division of Biology, Chemistry, and Material Science for instrument use.
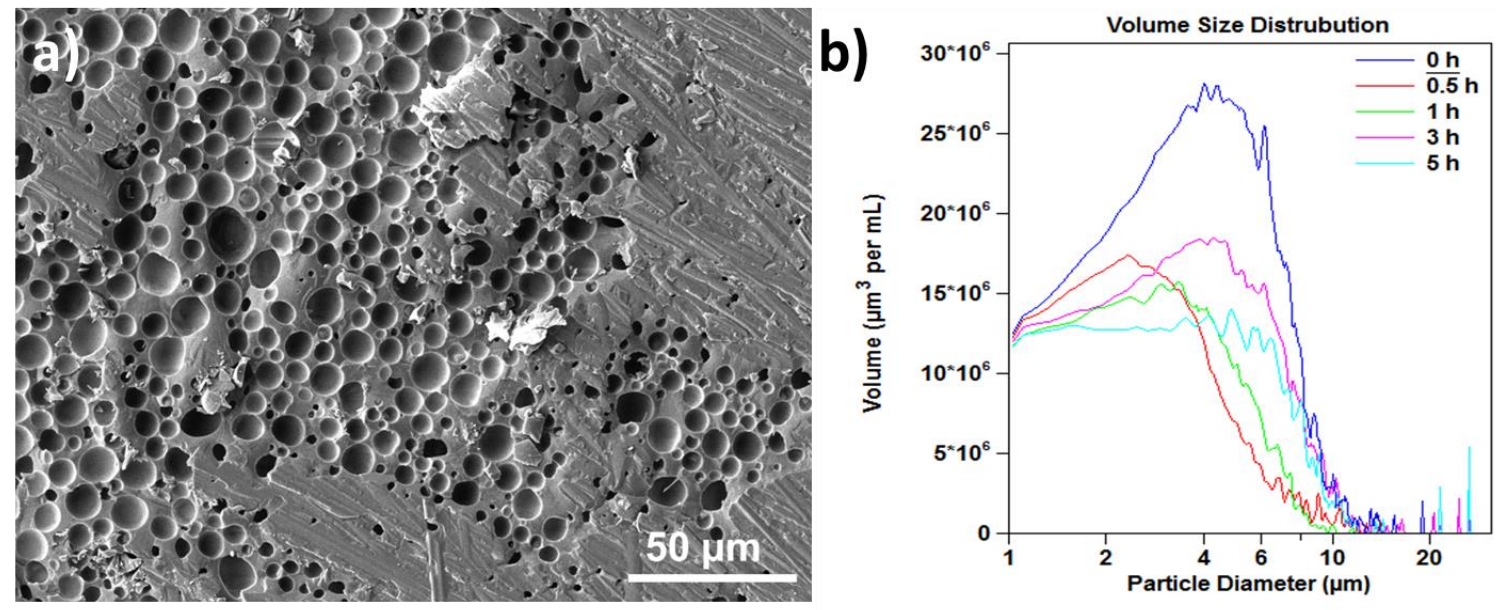

Figure 1. a) Representative cryo-SEM micrograph and b) Volume-based size distribution of the sulfur hexafluoride lipid microbubbles showing various range of size over time after agitation.

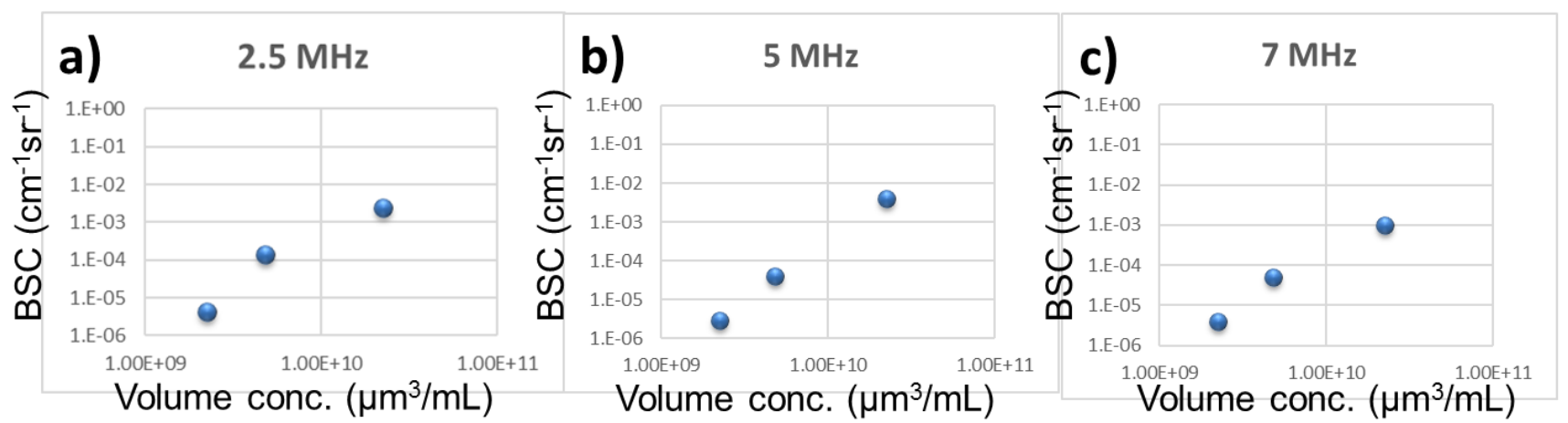

Figure 2. The correlation between bubble volume concentration and backscattering coefficient (BSC) at three clinically relevant frequencies: a) $2.5 \mathrm{MHz}$, b) $5 \mathrm{MHz}$, and c) $7 \mathrm{MHz}$. 